Nenckiu. Sieber: Verb.d. ein- u. zweibas. Fetts. etc. 147

\title{
Ueber die Verbindungen der ein- und zweibasischen Fettsäuren mit Phenolen;
}

von

M. Nenoki und N. Sieber.

\section{Mittheilung.}

Resorcin und Eisessig mit Chlorzink, als wasserentziehendem Mittel, erwärmt, vereinigen sich leicht zu einer neuen Verbindung, die ihrer Zusammensetzung und ihren Eigenschaften nach ein Dioxyacetophenon ist. Die Bildung dieser Substanz erfolgt nach der Gleichung: $\mathrm{C}_{6} \mathrm{H}_{8} \mathrm{O}_{2}+\mathrm{C}_{2} \mathrm{H}_{4} \mathrm{O}$. $=\mathrm{C}_{6} \mathrm{H}_{5}\left(\mathrm{C}_{2} \mathrm{H}_{3} \mathrm{O}\right) \overline{\mathrm{O}}_{2}+\mathrm{H}_{2} \mathrm{O}$. $\mathrm{Zu}$ ihrer Darstellung werden am zweckmässigsten 1,5 Gew.-Thle. Chlorzink in 1,5 Gew.Thln. Eisessig in der Wärme gelöst, der Flüssigkeit ein Gew.-Thl. Resorcin zugesetzt und im offenen Kolben auf dem Sandbade erhitzt. Bei $145^{\circ}-150^{\circ}$ kommt die Flüssigkeit in lebhaftes Sieden. Man entfernt die Flamme und lässt die Reaction sich auf dem Sandbade vollenden. Um die Bildung harziger Producte zu vermeiden, ist es nur nöthig darauf zu achten, dass die Temperatur nicht über $150^{\circ}$ steigt. Nach dem Erkalten mit Wasser versetzt, erstarrt die Schmelze zu einem, aus der neuen Verbindung bestehenden Krystallbrei. Während des Erhitzens nimmt die Flüssigkeit allmählich eine schöne, gelbrothe Farbe an. Wird sie über $150^{\circ}$ erhitzt, oder längere Zeit bei $150^{\circ}$ erhalten, so wird die Nüance eine tief rothe und durch weitere Einwirkung des Chlorzinks auf die entstandene Substanz entsteht ein harziges, in Alkalien mit violetter Farbe lösliches Product, wodurch natürlich die Ausbeute an der krystallinischen Substanz geringer wird.

Die abgeschiedenen, braun gefärbten Krystalle werden zur Entfernung des Chlorzinks mit verdünnter Salzsäure gewaschen. Sie lösen sich in Alkalien mit tief violetter Farbe auf. Durch längeres Kochen der alkalischen Lösung wird die Farbe offenbar in Folge allmähliger Zerstörung des Farbstoffs schmutzigbraun. Salzsäure fällt aus der verdünnten 


\section{Nencki und Sieber: Ueber die Verbindungen}

alkalischen Lösung die neue Verbindung unverändert aus. Die weitere Reinigung der im Wasser schwer löslichen Substanz geschah durch wiederholte Krystallisation aus verdünnter Salzsäure unter Zusatz von Thierkohle. Sie wird so in weissen rhombischen Blättchen oder Nadeln rein erhalten. Die an der Luft getrockneten Krystalle verloren im Exsiccator über Schwefelsäure nichts mehr an Gewicht, und ihre Elementaranalyse ergab folgende Zahlen:

$0,2259 \mathrm{Grm}$. der Substanz gaben 0,5211 Grm. $\mathrm{CO}_{2}$ und 0,1148 Grm. $\mathrm{H}_{2} \mathrm{O}$ oder in Proc. auf $\mathrm{C}$ und $\mathrm{H}$ berechnet: gefunden $\mathrm{C} 62,91 \%$ und $\mathrm{H} 5,64 \%$. Die Verbindung $\mathrm{C}_{8} \mathrm{H}_{8} \mathrm{O}_{3}$ enthält: $\mathrm{C} 63,15 \%$ und IH $5,26 \%$.

Im Capillarröhrchen schmilzt die Substanz bei $142^{\circ}$. Sie lässt sich nicht ohne Zersetzung destilliren. Bis zum Sieden erhitzt, geht anfangs bei $303^{\circ}-305^{\circ}$ ein farbloses Destillat über, das aber bald braun wird. Die Temperatur steigt allmählich bis über den Siedepunkt des Quecksilbers und der Retorteninhalt verharzt; auch bei vermindertem Luftdrucke war sie nicht ohne Zersetzung destillirbar. Im Vacuum des Hofmann'schen Apparates vergaste sie sich bei der Temperatur des siedenden Anilins nicht. Gegen Säuren und Alkalien ist die Substanz sehr beständig. Sie kann längere Zeit, ohne jede Veränderung, damit gekocht werden, und erst durch Schmelzen mit Kalihydrat wird sie zersetzt. Von kalter conc. Schwefelsäure wird sie gelöst und durch Wasserzusatz unverändert abgeschieden. Wie aus der empirischen Formel ersichtlich, kömnte die Substanz entweder als das bis jetzt unbekannte Monoacetylresorcin, oder, falls das Acetyl einen Wasserstoff des Benzolkerns substituirte, als ein Dioxyacetophenon angesehen werden. Wenn schon die Beständigkeit der Verbindung für die Annahme sprach, dass das Acetyl einen Benzolwasserstoff und nicht den hydroxylischen substituirte, so hat die Darstellung einer Acetylverbindung unserer Substanz und Vergleich derselben mit dem zuerst von Malin ${ }^{1}$ ) dargestellten Diacetylresorcin zu Gunsten dieser Annahme entschieden.

1) Ann. Chem. Pharm. 138, 78. 
der ein- u. zweibasischen Fettsäuren mit Phenolen. 149

Nachdem wir gesehen haben, dass sowohl Chloracetyl, als auch Essigsäureanhydrid auf unsere Substanz einwirken, wurde ein Gew.-Thl. der letzteren mit 3 Gew.-Thln. Essigsäureanhydrid am Rückflusskühler etwa eine Stunde lang im gelinden Sieden erhalten und sodann die Flüssigkeit aus einem Fractionirkölbchen destillirt. Nachdem Essigsäurehydrat, sowie das überschüssige Anhydrid übergingen, stieg der Quecksilberfaden rasch bis auf $303^{\circ}$, bei welcher 'Temperatur bis auf einen kleinen Rest fast Alles überging. Die bei $303^{\circ}$ übergegangene Fraction erstarrte sowohl in der Vorlage, wie im Kühlrohr krystallinisch und wurde zur Entfernung des ihm noch anhaftenden Essiganhydrids aus absolutem Alkohol zweimal umkrystallisirt. Der Schmelzpunkt der in feinen weissen Nadeln krystallisirenden Substanz lag bei $\mathbf{7 2}^{\circ}$, und ihre Elementaranalyse zeigte, dass sie mit dem Malin'schen Diacetylresorcin isomer ist.

$0,3379 \mathrm{Grm}$. der über $\mathrm{SO}_{4} \mathrm{H}_{3}$ bis zu constantem Gewichte getrockneten Substanz gaben 0,7634 Grm. $\mathrm{CO}_{2}$ and 0,1638 Grm. $\mathrm{H}_{2} \mathrm{O}$ oder in Proc.: $\mathrm{C} 61,61 \%$ und $5,38 \%$ H. Die Verbindung $\mathrm{C}_{6} \mathrm{H}_{4}\left(\mathrm{C}_{2} \mathrm{H}_{3} \mathrm{O}\right)_{2} \mathrm{O}_{2}$ enthält: C $61,85 \%$ und $5,15 \%$.

Malin erhielt das Diacetylresorcin durch Einwirkung von Chloracetyl auf Resorcin bei $100^{\circ}$ und beschreibt es als ein farb- und geruchloses, in Wasser unlösliches, nicht krystallisirendes Oel. Um uns von der Verschiedenheit unserer Acetylverbindung von dem Malin'schen Producte zu, überzeugen, haben wir reines, trocknes Resorcin mit dem dreifachen Gewichte Essigsäureanhydrid 2 Stunden lang am Rückflusskühler gekocht und sodann der fractionirten Destillation unterworfen. Sobald das Anhydrid überdestillirte, stieg die Temperatur rasch auf $272^{\circ}$, und zwischen $272^{\circ}$ bis $280^{\circ}$ ging der ganze Rest über. Diese Fraction wurde noch einmal rectificirt, wobei sie einen constanten Siedepunkt von $273^{\circ}$ (708 Mm. Bar.) hatte. Ihre Elementaranalyse zeigte, dass sie reines Diacetylresorcin von Malin war.

$0,2761 \mathrm{Grm}$. der Substanz gaben 0,6251 Grm. $\mathrm{CO}_{2}$ und 0,1263 Grm. $\mathrm{H}_{23} \mathrm{O}$, oder auf $\mathrm{C}$ and $\mathrm{H}$ berechnet: $61,74 \% \mathrm{C}$ und $5,08 \% \mathrm{H}$. Die Verbindung $\mathrm{C}_{6} \mathrm{H}_{4}\left(\mathrm{C}_{2} \mathrm{H}_{3} \mathrm{O}\right)_{2} \mathrm{O}_{2}$ enthält $\mathrm{C} 61,85 \%$ und $5,15 \% \mathrm{H}$.

$\mathrm{Da}$ die farb- und geruchlose Flüssigkeit auch nach 


\section{Nencki und Sieber: Ueber die Verbindungen}

wochenlangem Stehen im Exsiccator nicht krystallisirte, so ist nicht zu bezweifeln, dass sie von unserer Acetylverbindung verschieden und folglich das aus Eisessig und Resorcin erhaltene Product ein Dioxyacetophenon ist. Im Uebrigen giebt sowohl das Malin'sche Diacetylresorcin, als auch Resorcin und Essigsäureanhydrid beim Erwärmen mit Chlorzink ebenfalls unser Dioxyacetophenon. Bemerkenswerth ist es immerhin, dass auch durch längeres Kochen mit überschüssigem Essiganhydrid nicht die Diacetylverbindung des Dioxyacetophenons erhalten wurde.

Eine alkoholische Dioxyacetophenonlösung mit alkoholischem Natron versetzt bildet sofort ein in weissen Nadeln krystallisirendes Natronsalz, das aber an der Luft sich bald stark bräunt, und deshalb ergaben auch die. Natriumbestimmungen der mit Alkohol gewaschenen und im Exsiccator getrockneten Substanz keine übereinstimmenden Zahlen. Der Natriumgehalt des Salzes schwankte zwischen den beiden

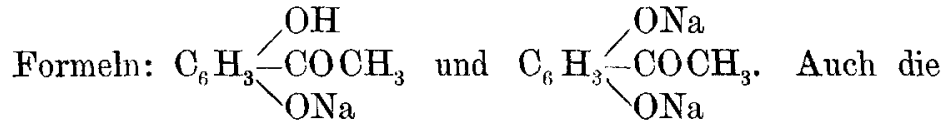
Acetylverbindung: $\mathrm{C}_{6} \mathrm{H}_{3} \stackrel{\mathrm{OH}}{\mathrm{COCH}} \underset{\mathrm{OOOCH}_{3}}{\mathrm{OCO}}$ mit alkoholischem Natron übergossen, giebt unter Erwärmen dasselbe Salz, gleichzeitig wird der erfrischende Geruch des Essigäthers bemerkbar. Charakteristisch ist die weinrothe Färbung, welche eine Spur des Dioxyacetophenons in Wasser gelöst mit Eisenchlorid giebt.

In der Absicht, eine Dioxybenzoësäure zu erhalten, haben wir das Dioxyacetophenon mit chromsaurem Kalium und Schwefelsäure oxydirt. Wir erhielten aber ausser geringen Mengen unveränderter Substanz nur noch Essigsäure. Mit dem dreifachen Gewichte Salpetersäure, spec. Gew. 1,4, übergossen, wird das Dioxyacetophenon in ein Mononitroproduct verwandelt. Die Reaction erfolgt ohne vorhergehendes Erwärmen. Nach beendigter heftiger Einwirkung wurde mit Wasser verdünnt, das krystallinisch abgeschiedene Nitroproduct abfiltrirt und aus 50proc. Alkohol umkrystallisirt, wobei 
der ein- u. zweibasischen Fettsïuren mit Phenolen. 151

sich die Substanz in schönen, langen, schwach gelb gefärbten Nadeln ausscheidet. Sie krystallisirt wasserfrei und schmilzt bei $142^{\circ}$. Die Elementaranalyse ergab folgende Zahlen:

$0,2701 \mathrm{Grm}$. der Substanz gaben 0,4791 Grm. $\mathrm{CO}_{2}$ und 0,0986 Grm. $\mathrm{H}_{2} \mathrm{O}$ oder $48,37 \% \mathrm{C}$ and $4,05 \% \mathrm{H}$.

$0,2999 \mathrm{Grm}$. gaben $19 \mathrm{Ccm}$. $\mathrm{N}$-Gas bei $16,5^{0} \mathrm{~T}$., $720 \mathrm{Mm}$. Bar. und über $20 \%$ Kalilauge, oder gefunden $6,99 \% \mathrm{~N}$.

Die Verbindung $\mathrm{C}_{6} \mathrm{H}_{2}\left(\mathrm{NO}_{2}\right)(\mathrm{OH})_{2}\left(\mathrm{C}_{2} \mathrm{H}_{3} \mathrm{O}\right)=\mathrm{C}_{8} \mathrm{H}_{7} \mathrm{NO}_{5}$ enthält: $\mathrm{C} 48,73 \%, \mathrm{H} 3,55 \%$ und $7,11 \% \mathrm{~N}$.

Die Nitrirung ist eine sehr glatte, und es scheint nur das Mononitroproduct zu entstehen. Die Elementaranalyse eines nur aus Wasser umkrystallisirten Productes ergab von der Theorie nur wenig abweichende Zahlen (gef. $\mathrm{C} 47,74 \%$, $\mathrm{H} 4,16 \%$ und $7,59 \% \mathrm{~N}$ ).

Aehnlich wie mit Essigsäure giebt Resorcin auch mit Buttersäure und Chlorzink erwärmt, ein neues Product, das aber flüssig ist. Wird zu einer Lösung von Chlorzink. in Ameisensäure Ręsorcin zugesetzt, so löst sich auch dieses zunächst darin auf. Auf dem Wasserbade erwärmt, nimmt die Flüssigkeit eine schön rothe Färbung an, und nach einiger Zeit erstarrt sie zu einer festen Masse. Wasserzusatz scheidet daraus ein rothes amorphes Pulver, das sich in Alkalien mit violetter Farbe löst und durch Säuren wieder gefällt wird. Andererseits verhält sich Pyrogallol, mit Chlorzink und Eisessig einen Augenblick zum Sieden erhitzt, genau so wie das Resorcin. Das durch Wasserzusatz abgeschiedene Product ist in heissem Wasser leicht, in kaltem weniger löslich, und wird durch Umkrystallisiren daraus, unter Zusatz von Thierkohle, leicht rein, in weissen, perlmutterglänzenden Blättchen erhalten. Die Elementaranalyse dieser Substanz, welche bei $168^{\circ}$ schmilzt, ergab zwar mit der Formel eines Trioxyacetophenons übereinstimmende Zahlen (gef. C 57,21\%, $\mathrm{H} 4,72 \%$; die Verbindung; $\mathrm{C}_{6} \mathrm{H}_{2}\left(\mathrm{C}_{2} \mathrm{H}_{3} \mathrm{O}\right)(\mathrm{OH})_{3}$ enthält: C 57,14\% und 4,76\% H). Da aber die procentische Zusammensetzung aller möglichen Acetylderivate des Pyrogallols die gleiche, wie die des Pyrogallols selbst ist, so müssen erst weitere Untersuchungen zeigen, ob die von uns erhaltene Substanz das nach der Analogie mit Resorcin erwartete 


\section{Nencki und Sieber: Ueber die Verbindungen}

T'rioxyacetophenon ist. Jedenfalls ist sie von dem bekannten Triacetyläther des Pyrogallols $\mathrm{C}_{6} \mathrm{H}_{3} \mathrm{O}_{3}\left(\mathrm{C}_{2} \mathrm{H}_{3} \mathrm{O}\right)_{3}$ verschieden.

Wie man sieht, lassen sich durch abwechselnde Anwendung eimbasischer Fettsäuren und Phenole mittelst Chlorzink eine ganze Reihe aromatischer Oxyketone bereiten. Wir sind mit der weiteren Bearbeitung der in dieser Reaction entstehenden Substanzen beschäftigt.

Die Leichtigkeit, mit welcher sich die Radicale der einbasischen Fettsäuren mittelst Chlorzink in den Benzolkern der Phenole einfügen lassen, veranlasste uns, auch das Verhalten der zweibasischen Fettsäuren, zunächst gegen Resorcin, zu prüfen. Ueber die Condensationsproducte aus Oxalsäure und Resorcin mittelst Schwefelsäure liegen Angaben von Claus und Andreae ${ }^{1}$, sowie Gukassiantz' vor. Bouchardat und Girard ${ }^{3}$ beschreiben in einem Patente. dass durch Einwirkung zweiatomiger Säuren, wie Oxalsäure, Milchsäure, Camphersäure u. s. w. auf Phenole und Diphenole bei $120^{\circ}-250^{\circ}$ Producte entstehen, welche unter der weiteren Einwirkung von unterchlorigsauren oder unterbromigsauren Alkalien Farbstoffe erzeugen, die aus der alkalischen Lösung durch Säuren abgeschieden werden können. In Bezug auf Bernsteinsäure hat schon vor längerer Zeit Malin ${ }^{4}$ ) die Beobachtung gemacht, dass Succinylchlorür mit Resorcin unter Salzsäureentwicklung ein gelbes Harz liefert, das in alkalischer Lösung durch den höchst intensiven grünen Dichroismus sich auszeichnete; Baeyer ${ }^{5}$ ), welcher den Versuch Malin's mit Bernsteinsäureanhydrid wiederholte, fand, dass in der That dabei eine, dem Fluoresceïn ausserordentlich ähnlich sehende Substanz sich bildet, so dass er nicht daran zweifelt, dass sie das Succineïn des Resorcins ist und vermuthlich nach folgender Gleichung entsteht:

$$
\mathrm{C}_{4} \mathrm{H}_{4} \mathrm{O}_{3}+2 \mathrm{C}_{6} \mathrm{H}_{6} \mathrm{O}_{2}=\mathrm{C}_{16} \mathrm{H}_{12} \mathrm{O}_{5}+2 \mathrm{H}_{2} \mathrm{O} \text {. }
$$

1) Ber. Berl. chem. Gres. 1877, S. 1305.

2) Das. 1878, S. 1184.

3) Liebig's Jahresb. 1877, S. 1234.

$\left.{ }^{4}\right)$ Ann. Chem. Pharm. 138, 79.

5) Ber. Berl. chem. Ges. 1871, S. 662 . 
der ein- u. zweibasischen Fettsäuren mit Phenolen. 153

Analysen, sowie genauere Untersuchung dieser fluorescirenden Substanz wurden jedoch von Baeyer nicht mitgetheilt.

Wir haben aus Bernsteinsäurehydrat und Resorcin durch Erhitzen sowohl mit Chlorzink, als auch mit concentrirter Schwefelsäure das Succinylfluorescein krystallinisch erhalten und eingehender untersucht.

Die Darstellung dieses Körpers mittelst conc. Schwefelsäure ist in Bezug auf die Ausbeute vortheilhafter, weil weniger harzige Producte als bei Anwendung von Chlorzink entstehen. 20 Grm. Resorcin, 13 Grm. Bernsteinsäure und $40 \mathrm{Grm}$. conc. $\mathrm{SO}_{4} \mathrm{H}_{2}$ werden in offenem Kolben auf $190^{\circ}-195^{\circ}$ etwa 1 Stunde lang erhitzt. Bei grösseren Quantitäten muss das Erhitzen entsprechend länger fortgesetzt werden. Die erkaltete Schmelze wird nun so lange mit verdünnter (etwa 3- bis 5 proc.) Salzsäure ausgekocht, als in einer Probe des Filtrates durch Bromwasser ein rother Niederschlag entsteht. Aus den ersten salzsauren Auszügen scheidet sich das Succinein in braungelben Krystallen aus, die unter dem Mikroskope lebhaft an die Formen der gefärbten Harnsäure erinnern. Die späteren Auszüge geben beim Erkalten keine Krystallisation mehr und werden zweckmässig mit Ammoniak genau neutralisirt. Das in Alkalien und verdünnter Salzsäure lösliche Fluoresceïn ist nämlich in Lösungen neutraler Salze so gut wie unlöslich, und scheidet sich beim Neutralisiren der sauren Lösung in gelben amorphen Flocken aus, welche aus heisser Salzsäure ebenfalls krystallinisch erhalten werden können. Die Ausbeute beträgt etwa $70-80 \%$ der theoretischen Menge, demn stets bleibt ein in verdünnter Salzsäure unlöslicher amorpher Rückstand, der sich in Alkalien mit rothbrauner Farbe löst, beim Verdünnen der Lösung stark fluorescirt und allem Anscheine nach identisch ist mit dem von Annaheim ') durch Erhitzen von Resorcin mit rauchender Schwefelsäure erhaltenen Producte. Die abfiltrirten Krystalle werden zunächst mit Wasser, sodann mit Alkohol (worin sich das Succinylfluoresceïn nur wenig löst) gewaschen

1) Ber. Berl. chem. Ges. 1877, S. 975. 


\section{Nencki und Sieber: Ueber die Verbindungen}

und schliesslich durch Umkrystallisiren aus verdünnter $\mathrm{HCl}$ unter Zusatz von Thierkohle rein erhalten. Die nur an der Luft getrockneten Krystalle haben die Zusammensetzung: $\mathrm{C}_{16} \mathrm{H}_{12} \mathrm{O}_{5}+3 \mathrm{H}_{2} \mathrm{O}$, wie aus folgenden Elementaranalysen ersichtlich:

0,2823 Grm. der an der Luft getrockneten Substanz gaben 0,5846 Grm. $\mathrm{CO}_{2}$ and 0,1412 Grm. $\mathrm{H}_{2} \mathrm{O}$ oder $56,46 \% \mathrm{C}$ und $5,55^{\circ}{ }_{0} \mathrm{H} . \quad 0,2965$ Grm. gaben 0,6148 Grm. $\mathrm{CO}_{2}$ und 0,1461 Grm. $\mathrm{H}_{2} \mathrm{O}$ oder $56,8^{\circ} \mathrm{O}$ und $5,47 \% \mathrm{H}$. Die Verbindung: $\mathrm{C}_{16} \mathrm{H}_{12} \mathrm{O}_{5}+3 \mathrm{H}_{2} \mathrm{O}$ enthält $\mathrm{C} 56,8 \%$ und $5,30 \% \mathrm{H}$.

Schon im Exsiccator verliert die Substanz ihr Krystallwasser und geht wahrscheinlich in das Fluoresceinnhydrat: $\mathrm{C}_{3} \mathrm{H}_{4}\left\langle{\mathrm{CO}-\mathrm{C}_{6} \mathrm{H}_{3}(\mathrm{OH})_{2}}_{2}^{\mathrm{CO} \mathrm{H}_{3}(\mathrm{OH})_{2}}\right.$ üb. Beim Liegen aber über $\mathrm{SO}_{4} \mathrm{H}_{2}$ ist der Verlust an Wasser geringer, als es die Formel des Fluorescë̈nhydrates verlangt; denn Analysen von über $\mathrm{SO}_{4} \mathrm{H}_{2}$ bis zu constantem Gewichte getrockneter Substanz ergaben für die Formel $\mathrm{C}_{16} \mathrm{H}_{14} \mathrm{O}_{6}$ zu niedrigen Kohlenstoffgehalt (gefunden $\mathrm{C} 62,05 \%$ und $5,0 \% \mathrm{H}$, ferner im gleichen Präparate $\mathrm{C} 62,05 \%$ und $5,2 \% \mathrm{H}$, berechnet für $\mathrm{C}_{16} \mathrm{H}_{14} \mathrm{O}_{6}$ C $63,5 \%$ und $4,63 \% \mathrm{H}$ ). Versucht man dann die Substanz im Luftbade zu trocknen, so verliert sie fortdauernd an Gewicht, ohne bei $130^{\circ}$, selbst bei $150^{\circ}$ constant zu werden. Dabei färbt sie sich stark braun. Die Analysen verschiedener, zwischen $110^{\circ}-150^{\circ}$ getrockneter Präparate ergaben zwischen den Formeln $\mathrm{C}_{18} \mathrm{H}_{14} \mathrm{O}_{8}$ und $\mathrm{C}_{18} \mathrm{H}_{13} \mathrm{O}_{4}$ liegende Zablen. Auch für das an der Luft getrocknete und aus absolutem Alkohol umkrystallisirte Fluoresceïn wurden noch der Formel $\mathrm{C}_{16} \mathrm{H}_{19} \mathrm{O}_{5}+2 \mathrm{H}_{2} \mathrm{O}$ am nächsten liegende Zahlen erhalten. Dass jedoch das Fluoresceïn der Bernsteinsäure durchaus analog dem der Phtalsäure zusammengesstzt ist, geht aus den Analysen des Tetrabromproductes (des Succinyleosins) hervor. Denn ähnlich wie die Fluorescenz des Succinylfluoresceïns in alkalischer Lösung an Schönheit dem Phtalsäurefluoresceïn fast gleich ist, so liefert es auch mit Brom einen schönen rothen Farbstoff, der die grösste Aehnlichkeit mit dem Baeyer'schen Eosin hat.

Die Darstellung des Succinyleosins ist eine sehr ein- 
fache. Kalt in verdünnter Salzsäure gesättigte Lösung wird mit Bromwasser, oder anch direct mit Brom, das man in dünnem Strahle zufliessen lässt, so lange versetzt, bis geringer Ueberschuss vorhanden, $\mathrm{d}$. $\mathrm{h}$. durch weiteren Zusatz keine Abscheidung des in Wasser und verdünnten Säuren ganz unlöslichen Tetrabromsuccinylfluoresceïns mehr erfolgt. Das in rothen amorphen Flocken am Boden des Gefässes abgeschiedene Product wird zunächst mit Wasser, hierauf mit Alkohol oder besser mit Aether gewaschen (in Alkohol und namentlich in Aether ist Succinyleosin nur wenig löslich), sodann in verdüunter Natron- oder Kalilauge gelöst, filtrirt und mit $\mathrm{HCl}$ gefällt. Ist die Substanz rein, so scheidet sie sich in sehr kleinen rothen Nädelchen aus. Die Elementaranalysen eines so erhaltenen und über $\mathrm{SO}_{4} \mathrm{H}_{2}$ getrockneten Präparates ergaben folgende Zahlen:

$0,3938 \mathrm{Grm}$. der Substanz gaben 0,4637 Grm. $\mathrm{CO}_{2}$ und 0,0565 Grm. $\mathrm{H}_{2} \mathrm{O}$ oder $32,11 \% \mathrm{C}$ und $1,59 \% \mathrm{H}$.

0,3106 Grm. der Substanz gaben 0,3893 Grm. AgBr, entsprechend $53,31 \%$ Br. Das Tetrabromsuccinylfluoresceïn $\mathrm{C}_{2} \mathrm{H}_{4}<\mathrm{CO}-\mathrm{C}_{6} \mathrm{HBr}_{2}<\mathrm{OH}$ $=\mathrm{C}_{16} \mathrm{H}_{8} \mathrm{Br}_{4} \mathrm{O}_{\overline{0}}$ enthält: $\mathrm{C} 32,0 \%, \mathrm{H} \mathrm{1,33 \%} \%_{0}$ und $53,33 \% \mathrm{Br}$.

Das Succinyleosin ist eine zweibasische Säure, doch sind es vorzugsweise die sauren Salze, welche sich durch ihre Krystallisationsfähigkeit auszeichnen. Die sauren Alkalisalze werden erhalten durch Auflösen des Farbstoffes in verdünntem Alkali und Zusatz von überschüssiger Essigsäure zu der siedend heissen Lösung. Beim Einkochen der Lösung scheiden sie sich in glänzenden braunrothen Krystallen aus, welche sämmtlich in Alkohol und Wasser schwer, und verhältnissmässig am leichtesten noch in Lösungen essigsaurer Alkalien löslich sind. Verdünnte Mineralsäuren scheiden die freie Säure wieder aus.

Das auf obige Weise erhaltene Kalisalz $\mathrm{C}_{16} \mathrm{H}_{7} \mathrm{E} \quad{ }_{5} \mathrm{~K}$ krystallisirt wasserfrei in braunrothen, glänzenden, rhombischen Nadeln. Die Elementaranalyse des an der Luft getrockneten Salzes ergab folgende Zahlen:

0,3196 Grm. der Substanz gaben 0,3534 Grm. $\mathrm{CO}_{2}$ und 0,0400 Gim. $\mathrm{H}_{2} \mathrm{O}$ oder $30,16 \% \mathrm{C}$ und $1,38 \% \mathrm{H}$. 
156 Nencki u. Sieber: Verb. d. ein-u. zweibas. Fetts. ete.

0,2278 Grm. gaben 0,2676 Grm. AgBr oder 49,98\% $\mathrm{Br}$.

$0,3202 \mathrm{Grm}$. gaben $0,0412 \mathrm{Grm} . \mathrm{SO}_{4} \mathbf{K}_{2}$ oder $5,78_{0}^{0} \mathbf{K}$.

Die Verbindung $\mathrm{C}_{10} \mathrm{H}_{7} \mathrm{Br}_{4} \mathrm{O}_{5} \mathrm{~K}$ enthält: $30,0 \% \mathrm{C}, 1,09 \%$ $50,14 \%$ Br und $6,13 \% \mathrm{~K}$.

Das auf gleiche Weise erhaltene saure Natrium- und Ammoniumsalz sind dem Kaliumsalze sehr ähnlich. Das erstere ist verhältnissmässig noch am leichtesten in heissem Wasser löslich. Das saure Calcium- und Bariumsalz entstehen durch Zusatz von Chlorcalcium resp. Chlorbarium zu der Lösung des sauren Natriumsalzes. Es sind in Wasser unlösliche, rothe krystallinische Niederschläge. Die Salze der schweren Metalle sind ebenfalls in Wasser unlöslich und amorph.

In Bezug auf die Färbekraft ist das Succinyleosin dem Phtaleosin gleich. Die Nüance ist nur ein wenig dunkler und auf Seide nicht so stark fluorescirend, als das Eosin von Baeyer.

Succinylfuoresceïn mit Salpetersäure von 1,4 spec. Gew. zum Sieden erhitzt, wird in ein schwach gelb gefärbtes, schön krystallisirendes 'Nitroproduct verwandelt, das aber kein Farbstoff ist und nicht mehr fluorescirt. Wir haben es nicht weiter untersucht. Wie schon oben erwähnt, geben ausser Oxalsäure und Bernsteinsäure nach den Angaben von Bouchardat und Girard auch andere zweiatomige Fettsüuren mit Resorcin fluorescirende Producte. Wir können noch hinzufügen, dass auch die halogensubstituirten einbasischen Fettsäuren, wie z. B. Chlor- oder Bromessigsäure, mit Resorcin und conc. $\mathrm{SO}_{4} \mathrm{H}_{2}$ ein Fluoresceïn giebt, welches in salzsaurer Lösung mit Brom behandelt in einen rothen Farbstoff verwandelt wird. Allem Anscheine nach verhalten sich diese Säuren ähnlich wie die zweiatomigen, d. h. es verbindet sich unter Austritt von $\mathrm{HCl}$ und $\mathrm{H}_{2} \mathrm{O}$ ein Molekül der Chloressigsäure mit 2 Molekülen Resorcin.

Bern, im December 1880. 